\title{
IMPORTANCIA DEL DESARROLLO DE COMPETENCIAS PARA EL EJERCICIO DEL PROFESIONAL EN CIENCIAS ECONÓMICAS EN UN CONTEXTO VICA
}

\author{
Oviedo, Raúl Ricardo \\ Gaibazzi, María Florencia \\ Leda, Ayelén Cecilia \\ Racca, Adriana Patricia \\ Siegenthaler, Bárbara Edith \\ Suñer, María Natalia
}

\section{Resumen:}

Este artículo resalta la importancia del desarrollo de competencias profesionales para el desempeño en un contexto caracterizado como volátil, incierto, complejo y ambiguo (VICA). Se sintetizan los resultados de un esfuerzo de cuatro años de trabajo en equipo, analizando la problemática desde cuatro perspectivas: alumnos, docentes, graduados y empresarios. Las competencias profesionales evaluadas son ordenadas en función de la importancia promedio atribuida, para luego analizar una a una su relevancia particular.

Palabras clave: Competencias - Profesionales - Ciencias Económicas - Contexto VICA

\begin{abstract}
:
This article highlights the importance of developing professional competences for performance in a context characterized as volatile, uncertain, complex and ambiguous (VUCA). The results of a four-year teamwork effort are synthesized, analyzing the problem from four perspectives: students, lecturers, graduates and entrepreneurs. The professional competences evaluated are ordered according to the average importance attributed, to then analyze their particular relevance one by one.
\end{abstract}

Key words: Competences - Professionals - Economic Sciences - VUCA Context

\section{INTRODUCCION}

El presente trabajo representa un cierre al Proyecto "Desarrollo de Competencias Profesionales para el desempeño efectivo en un entorno altamente complejo e inestable: La formación de profesionales competentes en Ciencias Económicas en la Universidad Nacional de Rosario", que se llevó adelante entre los años 2016 y 2019, sintetizando resultados obtenidos en el marco del mismo. Es asimismo un punto de partida para el 
Proyecto "Realidad Profesional del Licenciado en Administración: Representaciones, Expectativas, Formación y Ejercicio", que se extenderá desde 2020 hasta 2023.

Entonces, desde el recorrido realizado y su posterior orientación, bajo esta nueva producción de conocimiento, se direccionó un nuevo proyecto de investigación. Es decir, respondiendo a orientarse, para definir desde dónde se parte, puede repensarse y revisarse hacia dónde se dirige el enfoque de indagación en los años venideros, de labor.

Preguntarse desde el equipo, en su momento, respecto de cuáles son las competencias profesionales que los docentes, alumnos, graduados y empresarios priorizan como formación desde un contexto complejo, implicó e implica enunciar valores y prácticas laborales en juego que a menudo son implícitas. Es evidente que mejorar las condiciones de habilidades requeridas en un perfil profesional, lleva nociones de palabras tendenciosas, no dichas en lo cotidiano, de una labor, como progreso, eficacia, eficiencia, capacidad de respuesta, entre otras. Es decir, todas estas valoraciones convergen y se encausan en la necesidad imperiosa que se expresa en responder correctamente a las nuevas demandas tan cambiantes, variables y plurales, que el contexto VICA imparte.

Cabe aclarar a qué se hace referencia cuando se habla de un contexto VICA (Volátil, Incierto, Complejo y Ambiguo): remite a la extrema volatilidad de acontecimientos, que van cambiando permanentemente. Los tiempos para adaptarse se acortan cada vez más y las consecuencias de los hechos son cada vez menos previsibles. Puede decirse que "la única certeza que tenemos es que no hay certeza”. Los fenómenos son más complejos, lo que impide una clara descripción y conocimiento de los mismos para poder actuar en consecuencia. En el marco del presente contexto, toman especial relevancia las capacidades de adaptación y de cambio. Enfocadas desde la perspectiva humana de subsistencia la primera y de ventaja comparativa la segunda (Melamed, 2017, p.26).

En dicho contexto, los procesos laborales y personales de los sujetos como seres sociales remiten a que se experimente la vulnerabilidad, la fragilidad y la liquidez, si se toma a Bauman (2010) como un referente fundamental, respecto de la lectura sobre las implicancias sociales, económicas, ideológicas, políticas y subjetivas que provoca la época. Es decir, a pesar de la intencionalidad de esclarecer cuáles son las capacidades y habilidades profesionales que se repiten en el orden de lo prioritario, necesario o indispensable, el año 2020 en verdad es un claro e impensado ejemplo de cómo los criterios de validez que se intentan propiciar, resultan ser vertiginosamente mucho más cambiantes de lo que alguna vez alguien se podría imaginar. En estos tiempos se asiste a resolver profesionalmente sobre la misma inmediatez, y al mismo tiempo en un espacio 
en que las acciones de proyecciones futuras se encuentran absolutamente detenidas o a la espera de que algo, en algún momento, posibilite nuevos espacios de acción.

Mientras los requerimientos profesionales varían, es notable y constatable que, en tiempos de pandemia, las variaciones se precipitan. Podía resultar lejano sustituir el uso del documento escrito en papel y la presencialidad de los cuerpos, por la digitalización de la documentación y la virtualización de la presencialidad; hoy es posible decir que la realidad ha superado ampliamente a la ficción y a la imaginación, en la que se está inmerso.

No obstante, desde un trabajo compartido, se elige adoptar y avalar estos decires "el tiempo está de nuestra parte (...) somos nosotros quienes hacemos que las cosas sucedan" (Bauman, 2010, p.141).

Así como cada una de las competencias se orienta en sortear situaciones críticas, el uso de las competencias también da cuenta de un señalamiento prioritario en un universo desregulado. El progreso o la superación convergen y exaltan el plano de la individuación, la posibilidad de saber hacer en este proceso cambiante imparte que ellas sean la condición necesaria para metafóricamente poder profesionalmente 'surfear' o ‘bailar' bajo tamañas circunstancias.

La extrañeza y la paradoja son tendenciosas; sin embargo, frente a la crisis y el caos "una incesante conmoción, una inquietud y un movimiento constante, en vez de subvertir esta sociedad, sirven en realidad para fortalecerla" (Berman, 2010, p.90). En tanto las catástrofes pueden pensarse como oportunidades de desarrollo y de renovación, la desintegración actúa como una fuerza integradora.

Si bien en el contexto VICA la sensación puede ser de desconcierto y de fragmentación, Berman expone que esa paradoja es propia de la modernidad: solo quiere decir que la sociedad está viva.

En palabras de Hannah Arendt: "Los hombres no nacieron para morir, sino para innovar". (Beliz, 2017, p.58). La velocidad en la implementación de la transformación digital, su impacto disruptivo en general y en particular, tanto en el sector productivo como en el de servicio, se acelera a pasos agigantados; por lo tanto, debe uno aggiornarse y posibilitar el desarrollo de procesos de enseñanza y de aprendizaje acordes a los tiempos que corren. Que generen las competencias requeridas, en el marco de una educación democrática en donde los estudiantes puedan desarrollar mucho más sus capacidades 'blandas', tales como el liderazgo y el trabajo en equipo (Oppenheimer, 2018, p.224). 


\section{METODOLOGÍA}

Se realizó un estudio de alcance descriptivo, de corte transversal, con un enfoque cuantitativo.

Se aplicaron en las distintas poblaciones encuestas de opinión, por medio de cuestionarios autoadministrados, altamente estructurados, compuestos casi en su totalidad por escalas de valoración. En este trabajo, se analizarán los resultados de una pregunta particular, realizada a las cuatro poblaciones en estudio, referida a la importancia atribuida a una serie de competencias en relación al ejercicio del profesional en Ciencias Económicas. En todos los casos se evaluó el mismo listado de 16 competencias, con la misma escala de evaluación (escala de 1 a 5 , donde 1 significa 'Nada importante' y 5 'Muy importante'). Las competencias evaluadas son, en orden alfabético:

- Adaptación al Cambio

- Autorregulación Emocional

- Capacidad de Planificación

- Capacidad para Aprender

- Comunicación Efectiva

- Empatía

- Iniciativa

- Liderazgo Estratégico

- Manejo Constructivo del Conflicto

- Orientación al Cliente

- Pensamiento Creativo

- Pensamiento Crítico

- Responsabilidad Social

- Tolerancia a la Incertidumbre

- Trabajo en Equipo

- Visión sistémica

Las poblaciones en estudio son:

- Alumnos

- Docentes

- Graduados

- Empresarios 
En el caso de los alumnos, se encuestaron solamente aquellos que ya tenían su carrera definida, en las diferentes etapas de la misma. No se incluyó a los alumnos del Ciclo Introductorio a las carreras de Ciencias Económicas. En cuanto a los graduados, se optó por trabajar solamente con aquellos que se hubieran recibido en los cinco años previos a la realización del estudio. En docentes, no se establecieron restricciones, representando a los distintos años de las tres carreras. $Y$ en empresarios, se realizó una encuesta a representantes de empresas de diferentes rubros y tamaños, presentes en la ciudad de Rosario.

En el caso de alumnos y docentes, las encuestas fueron enviadas vía e-mail en archivo adjunto, mientras que en graduados y empresarios se envió una invitación de acceso a formularios cargados en la plataforma Survio. En las primeras tres poblaciones, se partió de bases provistas por la propia Facultad. El mail solicitando la colaboración con las encuestas fue reiterado hasta 3 veces. El mismo se envió a las bases completas, siendo la muestra, por tanto, autoseleccionada; esto es, se le envió a la población de alumnos y docentes y graduados, respondiendo quienes se prestaron a colaborar con la investigación. En la encuesta a empresarios, se trabajó con una base informal, construida por el propio equipo, al no contarse con un padrón confiable de empresas locales.

Se obtuvo un total de 394 encuestas de alumnos, 81 de docentes, 378 de graduados y 118 de empresarios.

Para la interpretación de los datos obtenidos, se utilizaron técnicas cuantitativas descriptivas, recurriéndose al paquete estadístico SPSS para su procesamiento.

\section{RESULTADOS}

A modo de establecer algún tipo de jerarquía o ranking general entre las competencias evaluadas desde las cuatro perspectivas, se calculó el promedio simple de las puntuaciones promedio para cada competencia desde cada perspectiva. Así, se obtuvo el ordenamiento ilustrado en el gráfico siguiente, si bien no se ilustra este valor en particular, que fue calculado solamente a los fines de generar un orden unificado. 


\section{Competencias más importantes en el ejercicio de la profesión}

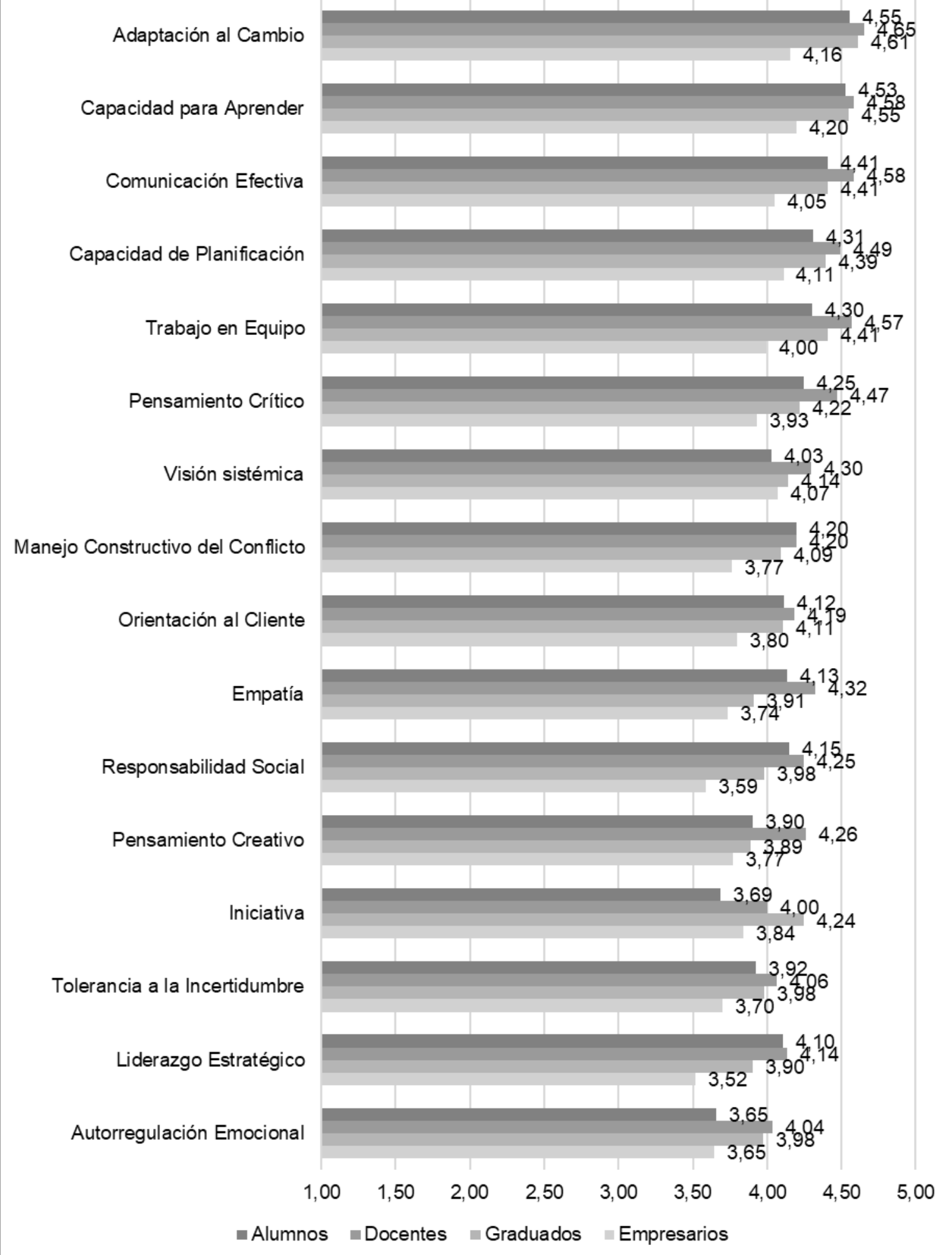


Así, se observa que las dos competencias con mayor importancia promedio en las cuatro perspectivas son Adaptación al Cambio y Capacidad para Aprender, ambas competencias íntimamente ligadas a la idea de desarrollo permanente del profesional en Ciencias Económicas, reforzada especialmente en un contexto volátil, incierto, complejo y ambiguo como el que estos profesionales deben enfrentar a diario. La Adaptación al Cambio está primera en las tres perspectivas académicas (alumnos, docentes y graduados), mientras que pasa al segundo desde la visión de los empresarios, que privilegian la Capacidad para Aprender (segunda para las otras perspectivas).

Les siguen como competencias esenciales: Comunicación Efectiva, Capacidad de Planificación y Trabajo en Equipo. Con sutiles diferencias, las tres ocupan el tercer, cuarto y quinto puesto en las perspectivas académicas, mientras que entre los empresarios se suma la Visión Sistémica en el cuarto lugar, por encima de Comunicación Efectiva y Trabajo en Equipo.

Más allá de las puntuaciones particulares, es destacable que en general todas las competencias evaluadas han sido consideradas importantes para el ejercicio profesional. En la escala de 1 a 5 utilizada, ninguna de las competencias obtiene un valor promedio por debajo de 3,5 en ninguna de las perspectivas - de hecho, para los graduados, ninguna baja de 4 puntos promedio. Esto da cuenta de cómo, si bien hay competencias consideradas más importantes que otras, todas las competencias directivas evaluadas son consideradas necesarias para un desempeño efectivo.

Así también, a diferencia de las competencias consideradas más importantes, no hay tanto acuerdo entre las diferentes perspectivas respecto a cuáles serían las menos importantes. Podrían destacarse como las menos valoradas en general Autorregulación Emocional y Liderazgo Estratégico.

A continuación, se abordarán una a una las competencias profesionales consideradas en el proyecto, comentando su importancia para el ejercicio del profesional en Ciencias Económicas.

\subsection{Adaptación al Cambio}

Adaptación al Cambio
Alumnos

4,55
Docentes

4,65
Graduados Empresarios

4,61

4,16

En un contexto como el que se atraviesa en la actualidad, altamente volátil, incierto, complejo y ambiguo (VICA) no cabe duda que la capacidad de adaptarse a los cambios 
es fundamental para la supervivencia de las organizaciones y para el desarrollo profesional de los graduados en Ciencias Económicas. Por ello, no es de extrañar que la Adaptación al Cambio sea la competencia más valorada en el promedio de las cuatro perspectivas, siendo la más valorada por alumnos, docentes y graduados y la segunda para los empresarios, solo por debajo de la Capacidad de Aprender.

Para los profesionales, es indispensable poder adaptarse a los cambios que como se mencionó antes son cada vez más profundos e impredecibles. Esta competencia aporta flexibilidad en el comportamiento tan necesaria para cumplir con los objetivos fijados y alcanzar un liderazgo empresarial y profesional.

\subsection{Capacidad para Aprender}

Capacidad para Aprender

\section{Alumnos}

4,53
Docentes

4,58
Graduados Empresarios

4,55

4,20

Puede precisarse que esta competencia es altamente valorada por docentes, alumnos y graduados, y ligeramente menos considerada por los empresarios.

Esta competencia se enmarca en las competencias reconocidas como genéricas o transversales, porque incluye la capacidad de análisis y de síntesis de visiones globales y de aplicación de los saberes en la práctica, en tanto se deben asumir responsabilidades, actuar con criterios propios, evaluar y comprender resultados.

Esta capacidad considerada en menor escala por el empresariado, quizás remite a que las expectativas que se depositan en el experto contratado, son que su labor instruya a otros y no en sentido contrario en tanto las expectativas suponen un rendimiento superior.

\subsection{Comunicación Efectiva}

Comunicación Efectiva

\section{Alumnos}

4,41

\section{Docentes}

4,58

\begin{tabular}{cc|} 
Graduados & Empresarios \\
4,41 & 4,05 \\
\hline
\end{tabular}

Es la tercera competencia con mayor importancia promedio en las cuatro perspectivas. Desde la visión de los alumnos y de los docentes, comunicación efectiva se posiciona en el tercer lugar; según el análisis de los graduados, la misma ocupa el cuarto lugar y desde la perspectiva de los empresarios, aquella se encuentra en el quinto puesto de preferencia. 
Puede decirse que esta competencia es una herramienta indispensable y determinante para las organizaciones (públicas, privadas y del tercer sector), porque a través de ella el recurso humano puede conocer con claridad cuáles son las metas y objetivos a seguir dentro de la organización, generando un dinamismo particular orientado a cumplir los objetivos propuestos en un ámbito de mayor confianza y, en caso del ámbito empresarial, impactando directamente sobre la productividad de la empresa (Raffino, 2020).

\subsection{Capacidad de Planificación}

Capacidad de Planificación

\section{Alumnos}

4,31

\section{Docentes} 4,49
Graduados Empresarios 4,39
4,11

La capacidad de planificación se encuentra en promedio dentro de las 4 primeras más relevantes para las cuatro perspectivas: alumnos, docentes, graduados y empresarios, demostrando la importancia de la misma y su incidencia en el recorrido académico y desarrollo profesional.

"La capacidad de planificación es el proceso mental que nos permite seleccionar las acciones necesarias para alcanzar una meta, decidir sobre el orden apropiado, asignar a cada tarea los recursos cognitivos necesarios y el establecer el plan de acción adecuado" (CogniFit, s.f.).

Tal como se expresa en la definición ut-supra, se puede vislumbrar la envergadura de la misma para el desarrollo y ejercicio profesional en el contexto VICA, en el cual además de planificar se requiere replanificar ante los cambios permanentes que se presentan y la adaptabilidad necesaria que se requiere para redefinir el plan de acción y alcanzar las metas establecidas.

\subsection{Trabajo en Equipo}

Trabajo en Equipo

\section{Alumnos}

4,30
Docentes

4,57
Graduados Empresarios 4,41 4,00

Dado el puntaje promedio de todos los actores involucrados, ocupa el quinto lugar dentro de las competencias más valoradas. Sin embargo, es interesante destacar, que son los formadores de la disciplina quienes ponderan más esta competencia; probablemente ya que es una característica que es sumamente evidenciable en el proceso de enseñanzaaprendizaje. Por otra parte, son quienes demandan al profesional (empresarios) quienes 
menos ponderan esta característica, lo cual probablemente esté asociado a la visión del perfil del trabajo profesional como consultor.

Más allá de estas pequeñas diferencias en los puntos de vista de los actores, en un contexto VICA, especialmente en la crisis sanitaria actual, el trabajo en equipo se vio modificado en sus posibilidades reales por no poder realizar la reunión, en la mayoría de los casos, de forma física. En consecuencias, se destacó la habilidad para trabajar en equipo de forma "virtual", con las características que esto implica, entre ellas: posibilidades de conectividad de cada integrante, mayor división de las tareas, coordinación y, por sobre todo, cooperación con aquellos quienes, por un contexto determinado en sus hogares/familias, o por su salud, no pueden asistir a sus lugares de trabajo o cumplir al $100 \%$ con las exigencias pautadas.

\subsection{Pensamiento Crítico}

\section{Pensamiento Crítico}

Alumnos

4,25
Docentes

4,47
Graduados Empresarios 4,22
3,93

El pensamiento crítico ocupa el sexto lugar en la valoración promedio de las cuatro perspectivas, siendo más valorados por los docentes que por el resto de los grupos encuestados. Sin embargo, no es muy distante la valoración dada por los distintos claustros, ya que ocupa el sexto lugar tanto en alumnos como en docentes y el séptimo para los empresarios y los graduados.

En el artículo "El Pensamiento Crítico desde la Perspectiva de los Docentes Universitarios" se trascribe la definición elaborada por Robert Sternberg, quien escribía que "el pensamiento crítico son los procesos, estrategias y representaciones que la gente utiliza para resolver problemas, tomar decisiones y aprender nuevos conceptos" (Bezanilla Albisua et. al., 2018).

Dicha competencia es esencial para el desarrollo y la empleabilidad profesional. El articulo citado también recoge, basado en Kurland, el criterio de que "la clave no está en tener la razón, sino en tener en cuenta todas las posibilidades y confiar en la razón más que en la emoción, para no permitir que nuestros prejuicios sesguen nuestras decisiones" (ibid.). Desde esta perspectiva el pensamiento crítico es necesario para la creatividad, la innovación y la resolución de problemas. 


\subsection{Visión sistémica}

\begin{tabular}{|ccccc|}
\hline Visión sistémica & Alumnos & Docentes & Graduados & Empresarios \\
& 4,03 & 4,30 & 4,14 & 4,07 \\
\hline
\end{tabular}

Esta competencia se ubica en el séptimo puesto en cuanto a la valoración promedio entre perspectivas. Aquí es destacable que, a diferencia de la mayoría de las competencias analizadas, la ponderación del empresario es mayor que la del alumno. Teniendo en cuenta que la visión sistémica implica un conjunto de elementos interrelacionados, como visión antagónica de compartimentos estancos; dentro de la organización implica funcionar de forma tal que las áreas estén relacionadas entre sí y que se cumplan los objetivos planteados por el ápice estratégico de la organización (empresario), de forma transversal; sin que las mismas actúen de forma aislada. Este hecho explicaría la mayor valoración del empresario.

En este contexto VICA, el trabajo interdisciplinario, que implica la visión sistémica, es de importancia para el cumplimiento y seguimiento de los objetivos planteados por la organización con el desafío de la integralidad en el aislamiento. Una clara visión sistémica, con áreas interrelacionadas ante un contexto de aislamiento como el actual, sin dudas facilita el funcionamiento organizacional y la adaptación al medio.

\subsection{Manejo Constructivo del Conflicto}

\begin{tabular}{|ccccc|}
\hline \multirow{2}{*}{ Manejo Constructivo del Conflicto } & Alumnos & Docentes & Graduados & Empresarios \\
& 4,20 & 4,20 & 4,09 & 3,77 \\
\hline
\end{tabular}

Es la octava competencia con mayor importancia promedio en las cuatro perspectivas. Desde la visión de los alumnos, manejo constructivo del conflicto se encuentra en el séptimo lugar; para graduados, se posiciona en el décimo puesto; y desde el análisis de los docentes y empresarios, la misma ocupa el décimo primer lugar de preferencia.

Se puede referenciar a esta competencia como aquella vinculada a la existencia de decisiones compartidas, mutuamente beneficiosas y productivas, en un contexto de ideas divergentes. Esta situación puede presentarse cuando los miembros de una organización presentan diferencias en cuanto a las tareas a realizar o a las prioridades, abriéndose un espacio para la discusión y el debate, con la finalidad de conseguir soluciones integrales. Esta instancia de intercambio demuestra la prioridad que las partes atribuyen tanto al proceso como al resultado (Harvard Business Review, 2017). 


\subsection{Orientación al Cliente}

Orientación al Cliente
Alumnos

4,12
Docentes

4,19
Graduados Empresarios

4,11

En cuanto al desarrollo de la capacidad sobre la orientación al cliente, se observa una importancia moderada ya que en promedio se encuentra en un noveno lugar dentro de un total de 16 capacidades, destacándose que en la encuesta el sector empresario fue el que menor puntaje le asignó.

"La Orientación al Cliente es más que una habilidad, es una forma de pararse frente al negocio. Es una posición que se adopta al pensar en el desarrollo del negocio, en toda su cadena de valor, desde la comunicación de la oferta o la logística hasta la atención post venta. Es ponerse en el lugar de quien 'compra' y hacer ese viaje/recorrido por la oferta y servicios de la empresa, para ver, con ojos de cliente, si es posible cumplir con sus expectativas y además aportar valor" (OBS Business School, s.f.).

Tal como se expresa en la definición ut-supra, se puede vislumbrar la importancia de la misma para el ejercicio profesional en el marco del contexto VICA, en el cual el cliente es la estrella, se encuentra en el centro de la escena. Por lo cual primero se debe definir el para quién, conocer sus hábitos, necesidades, requerimientos, ecosistema funcional, etc. y luego recién comenzar a pensar en el qué y el cómo.

\subsection{Empatía}

\begin{tabular}{|ccccc|}
\hline \multirow{2}{*}{ Empatía } & Alumnos & Docentes & Graduados & Empresarios \\
& 4,13 & 4,32 & 3,91 & 3,74 \\
\hline
\end{tabular}

Las encuestas posicionan a esta competencia como de menor interés que las anteriormente enunciadas, en el puesto diez. $Y$ quienes mayormente le restan cierto valor, por los resultados arrojados, son los empresarios.

La empatía es una habilidad que infiere en la capacidad de comprender a los demás, e interferir asertivamente sobre el pensamiento, en los estados de ánimo, y sus motivaciones. Se requiere respeto, neutralidad, escucha y observación, favoreciendo una comunicación positiva, pero también su equilibrio es frágil, si se involucra demasiado emocionalmente, con cuestiones que deben resultar distantes.

Entonces, así definida, en sí misma puede revisarse, ya que lo más dificultoso de esta habilidad es la posibilidad de ejercer un equilibrio entre la atención personal que debe 
brindarse a los compañeros de trabajo, y la distancia que debe tomarse, para no involucrarse desmedidamente en cuestiones impropias.

Los empresarios respecto de esta habilidad quizás son cautos o reacios a la hora de favorecer los 'amiguismos' o las 'complicidades' laborales, en tanto que el equilibrio en las relaciones humanas siempre es complicado.

\subsection{Responsabilidad Social}

Responsabilidad Social

\begin{tabular}{cccc|} 
Alumnos & Docentes & Graduados & Empresarios \\
4,15 & 4,25 & 3,98 & 3,59 \\
\hline
\end{tabular}

Esta competencia no es de las más ponderadas. Es interesante destacar que, dentro de los actores encuestados, quienes más la ponderan son los docentes, probablemente influenciados por el rol formativo que tienen en la sociedad en su conjunto. Por otro lado, son los empresarios quienes menos ponderan esta competencia, que son los responsables de la elección del profesional que los asesore/trabaje en sus organizaciones.

Queda por demás de notoria la necesidad de responsabilidad social en un contexto VICA, especialmente en esta crisis sanitaria, donde una conducta responsable, ya no es solo por el cuidado propio, sino por un otro que se vincula socialmente con uno y que comparte el territorio donde interactúan. La responsabilidad social "implica un compromiso que debemos asumir todos; no solo la empresa privada. A partir de identificar nuestras principales carencias y problemáticas, y de contribuir a resolverlas desde el lugar que nos toca actuar, ya sea como empresa, como ciudadano, consumidor o usuario, como sociedad, como gobierno local, como universidad, como ONG, como emprendedor, como sindicato, como cooperativa, realizando entre todos acciones socialmente responsables, que pueden ser complementarias o convergentes entre sí" (Minicelli, 2013, p.184) En definitiva, la empresa no está exenta de esta conceptualización; al contrario, se vuelve cada vez más necesaria y fundamental dentro de este contexto.

\subsection{Pensamiento Creativo}

Pensamiento Creativo
Alumnos

3,90
Docentes

4,26
Graduados Empresarios

3,89
3,77 
De Bono (2004) hace hincapié en el pensamiento creativo como una destreza que se puede aprender y que es indispensable para que las organizaciones se adelanten y se adecuen a sus contextos más allá de las pautas conocidas o los caminos establecidos.

Si bien, como manifiesta el autor mencionado, es notoria la necesidad del pensamiento creativo para la solución de problemas y la generación de ideas que se traduzcan en innovaciones necesarias para hacer frente a este entorno tan convulsionado y cambiante, históricamente el pensamiento lógico se ha impuesto por sobre el creativo, y a pesar de ser complementarios se han visto como competidores en detrimento de este último.

Nuestro sistema educativo también se orienta claramente al pensamiento lógico y reserva un lugar marginal a la creatividad. De allí que no llama la atención que el Pensamiento Creativo ocupe el doceavo lugar en la valoración promedio de las cuatro perspectivas, ocupando el mejor lugar entre los docentes (noveno lugar), décimo lugar para los empresarios, decimocuarto para los alumnos y el último lugar para los graduados.

\subsection{Iniciativa}

\begin{tabular}{|ccccc|}
\hline \multirow{2}{*}{ Iniciativa } & Alumnos & Docentes & Graduados & Empresarios \\
& 3,69 & 4,00 & 4,24 & 3,84 \\
\hline
\end{tabular}

Es la décima tercera competencia con mayor importancia promedio en las cuatro perspectivas. Desde la visión de los graduados, se encuentra en el sexto lugar; para los empresarios, se posiciona en el octavo puesto; desde el análisis de los alumnos, la misma se encuentra en el décimo quinto puesto y, para los docentes, aquella ocupa el décimo sexto lugar de preferencia.

Según Henry Fayol, esta competencia representa a uno de los 14 principios de la administración moderna. Para el científico, una de las prioridades de toda organización es alentar al recurso humano a realizar sus propios planes e intentar ejecutarlos al interior del organismo. Esta situación da como resultado la elevación de la autoestima y la seguridad de las partes (sobre todo, de los empleados), generando plena satisfacción por el desarrollo de sus capacidades y generando, además, el éxito de la organización (Riquelme, 2014).

\subsection{Tolerancia a la Incertidumbre}

\begin{tabular}{|ccccc|}
\hline \multirow{2}{*}{ Tolerancia a la Incertidumbre } & Alumnos & Docentes & Graduados & Empresarios \\
& 3,92 & 4,06 & 3,98 & 3,70 \\
\hline
\end{tabular}


En cuanto al desarrollo de la capacidad sobre la tolerancia a la incertidumbre, se le atribuye una importancia baja ya que en promedio se encuentra en el décimo cuarto lugar dentro de un total de 16 capacidades, destacándose que en la encuesta el sector empresario fue el que menor puntaje le asignó.

"La tolerancia a la incertidumbre es precisamente esa capacidad para lidiar con la falta de certezas y de soluciones inmediatas" (Sánchez, 2018).

Tal como se expresa en la definición ut-supra, se puede vislumbrar la importancia de la misma para el desarrollo y ejercicio profesional en el marco del contexto VICA, en el cual la única certeza que existe es que no hay certezas, lo único permanente es el cambio y el mundo es cada vez más complejo. Por lo dicho anteriormente si bien esta competencia según las encuestas no manifiesta una considerada significación, es muy necesaria y de gran valor en la actualidad.

\subsection{Liderazgo Estratégico}

\section{Liderazgo Estratégico}

Alumnos

4,10
Docentes

4,14
Graduados Empresarios 3,90 3,52

Esta competencia es otra de las que ocupan los últimos lugares dentro del ranking de competencias. Luego de los docentes, quienes más la ponderaron fueron los alumnos siguiéndoles los graduados. Quienes están más interesados en llevar la estrategia a la organización serían los empresarios; sin embargo, le otorgan parcialmente ese rol al profesional en Ciencias Económicas.

Siguiendo a Santiago Lazzati, el liderazgo es: "el proceso por el cual una persona influye en otras para que se encaminen hacia el logro de los objetivos comunes" (2015, p.22); en la organización sería la estrategia, y es probablemente esa influencia que entienda el empresario debe ser ejercida por su persona (especialmente en las pymes).

En el actual contexto de crisis sanitaria, es un gran desafío poder seguir cumpliendo con las tareas encaminadas hacia el logro de los objetivos. Al igual que en todo contexto cambiante, el proceso de influencia es fundamental para el logro de los objetivos/estrategias organizacionales y, por tanto, la necesidad de liderazgo se vuelve mayor. 


\subsection{Autorregulación Emocional}

Autorregulación Emocional

Alumnos

Docentes

Graduados Empresarios

3,65

4,04

3,98

3,65

Esta es la capacidad de expresar las emociones de forma adecuada, en tanto implica gestionar bien los conflictos y las situaciones tensas. Ser consciente de las propias emociones y el impacto que las mismas producen en los demás es el factor determinante. En la actualidad otro modo de enunciar esta habilidad seria inteligencia emocional.

Representa la última competencia valorada, por orden de relevancia en las encuestas. Preguntarse qué factores o circunstancias generaran una menor tendencia, sobre todo en los alumnos y empresarios para su elección, no resulta simple extraer una consideración al respecto. Tal vez algunas competencias interceden mucho más en la eficiencia o la eficacia profesional, a diferencia de esta que representa un alto componente psicológico en sí mismo, que no se traduce directamente sobre un trabajo eficazmente hecho. Es decir. el graduado puede ser impecable en todas las presentaciones laborables a pesar de manifestarse como una persona ansiosa, temerosa, conflictiva o hiperactiva.

\section{CONCLUSIONES}

Sin dudas la Humanidad, a partir de la pandemia generada por el COVID-19, está atravesando un momento de quiebre histórico, donde las palabras que definen al contexto VICA (Volatilidad, Incertidumbre, Complejidad y Ambigüedad) adquieren otra gravedad y otra dimensión. Estamos en un contexto de crisis profunda y caos que llevará a la redefinición de muchos paradigmas económicos, políticos y sociales.

A raíz de los resultados obtenidos en este trabajo de investigación, puede concluirse que tanto los alumnos, los docentes, los graduados como así también, los empresarios, prestan vital importancia a aquellas competencias que son mayormente requeridas en un contexto VICA, como son Adaptación al Cambio y Capacidad para Aprender. Puede decirse que, la adaptabilidad a múltiples escenarios distintos, como así también, la aptitud para adquirir nuevos conocimientos (que se actualizan constantemente) son vinculados por los actores previamente mencionados con la eficiencia en términos de productividad y con el efectivo desarrollo profesional.

Por otro lado, los encuestados también hicieron hincapié, aunque en menor medida, sobre aquellas competencias que implican la organización y planificación entre varios sujetos, con especial énfasis al desenvolvimiento profesional que también contemple una mirada 
sistémica y global frente a los cambios. Puede decirse que, para aquellos, el desarrollo de las aptitudes profesionales implica además el intercambio de ideas y la manifestación de diversas opiniones, pero cuya finalidad principal sea encontrar puntos en común y planificar objetivos concretos.

Sin embargo, llama la atención la baja valoración de dos competencias esenciales para hacer frente a este contexto: la Empatía (que ocupa en promedio el décimo lugar) y el Pensamiento Creativo (duodécimo lugar), tan necesario para dar nuevas respuestas a realidades disruptivas en las que los métodos y los paradigmas tradicionales se han agotado.

Finalmente, cabe aclarar que todos los grupos de encuestados dan una gran importancia a la formación en competencias y mayormente existe acuerdo en la valoración que les dan a las mismas.

\section{FUENTES}

El gráfico y las tablas son de elaboración propia en base al relevamiento realizado.

\section{REFERENCIAS BIBLIOGRÁFICAS}

\section{Libros}

Bauman, Z. (2010) Modernidad Líquida. Buenos Aires. Fondo de Cultura Económica.

Beliz, G. (2017) Robotlución. CABA. Planeta.

Berman, M. (2010) Todo lo sólido se desvanece en el aire. La experiencia de la modernidad. Buenos Aires. Siglo XXI Editores.

De Bono, E. (2004) El pensamiento creativo. México. Paidós.

Lazzati, S. (2015) El Gerente. Estratega y líder del cambio. Más allá de la gestión operativa. CABA. Granica.

Melamed, A. (2017) El futuro del trabajo y el trabajo del futuro. CABA. Planeta.

Minnicelli, A. (2013) Pequeño Diccionario de la Responsabilidad Social. CABA. Fundación Observatorio de Responsabilidad Social.

Oppenheimer, A. (2018) Sálvese quien pueda. CABA. Debate.

\section{Artículos}

Bezanilla Albisua, M. J.; Poblete Ruiz, M.; Fernández Nogueira, D.; Arranz Turnes, S.; Campo Carrasco, L. (2018) El Pensamiento Crítico desde la Perspectiva de los Docentes Universitarios. Estudios pedagógicos. Vol.44, no.1. Valdivia. Disponible 
en: https://scielo.conicyt.cl/scielo.php?script=sci_arttext\&pid=S071807052018000100089 [Fecha de consulta: 07 de Septiembre de 2020].

Casanellas Chuecos, M., Camos Ramio, M.; Medir Tejado, LL.; Montolio Estivil, D. Sibina, Tomas, D.; Sole Catalana, M.; Sayos Santigosa, R. (s.f.) Capacidad de aprendizaje. Indicaciones dirigidas al alumnado para adquirir la competencia en el grado de Gestión y Administración Pública. Gestión y Administración Pública (GAP). Facultad de Derecho. Universidad de Barcelona. España. Disponible en: http://diposit.ub.edu/dspace/bitstream/2445/58464/1/Capacidad\%20de\%20aprend izaje\%20\%20-\%20Protocolo\%20estudiantes.pdf [Fecha de consulta: 04 de Septiembre de 2020].

CogniFit (s.f.) Planificación: Neuropsicología de las funciones ejecutivas. Disponible en: https://www.cognifit.com/es/habilidad-cognitiva/planificacion [Fecha de consulta: 06 de Septiembre de 2020].

De Molina, C. (2015) La empatía como competencia profesional. Ingenia. Disponible en: https://ingenia.es/actualidad/conocimiento/la-empatia-como-competenciaprofesional/\#: :text=La\%20empat\%C3\%ADa\%20es\%20una\%20habilidad,decisio nes\%20y\%20formas\%20de\%20actuar [Fecha de consulta: 04 de Septiembre de 2020].

Harvard Business Review (2017) Cómo manejar conflictos constructivos y destructivos. Dirige Hoy. Disponible en: https://dirigehoy.info/free/1_minute_tips/toma-dedecisiones/como-manejar-conflictos-constructivos-y-destructivos/ [Fecha de consulta: 31 de Agosto de 2020].

OBS Business School (s.f.) Competencias Digitales: Orientación al Cliente. Disponible en: https://obsbusiness.school/es/blog-investigacion/social-media/competenciasdigitales-orientacion-al-cliente [Fecha de consulta: 06 de Septiembre de 2020].

Raffino, M. E. (2020) Concepto de Comunicación efectiva. Disponible en: https://concepto.de/comunicacion-efectiva/ [Fecha de consulta: 31 de Agosto de 2020].

Riquelme, M. (2014) Los 14 principios de Henry Fayol. Web y Empresas. Disponible en: https://www.webyempresas.com/los-14-principios-de-henry-fayol/ [Fecha de consulta: 31 de Agosto de 2020]. 
Sánchez, E. (2018) La tolerancia a la incertidumbre, una clave para vivir. Disponible en: https://lamenteesmaravillosa.com/la-tolerancia-a-la-incertidumbre-una-clave-paravivir/ [Fecha de consulta: 06 de Septiembre de 2020].

Vergara, C. (2015) 5 competencias esenciales para ser emocionalmente inteligente. Actualidad en Psicología. Disponible en: https://www.actualidadenpsicologia.com/competencias-inteligenciaemocional/\#: :text=La\%20autorregulaci\%C3\%B3n\%20es\%20la\%20competencia, de\%20situaciones\%20tensas\%200\%20dif\%C3\%ADciles [Fecha de consulta: 04 de Septiembre de 2020]. 